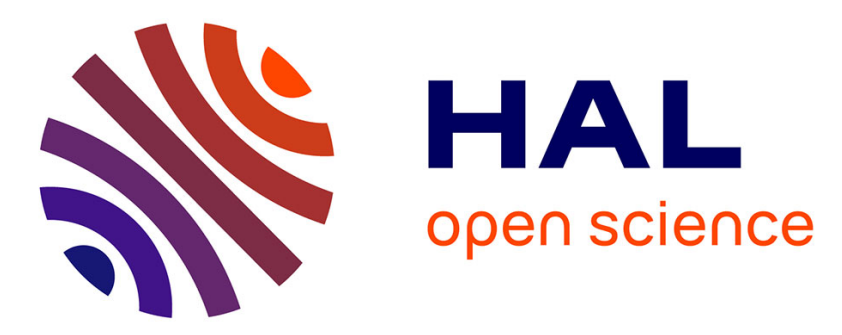

\title{
Maxwell-Boltzmann periodic structures by point defects in ionic crystals
}

\author{
M. Georgiev, N. Martinov, D. Ouroushev
}

\section{To cite this version:}

M. Georgiev, N. Martinov, D. Ouroushev. Maxwell-Boltzmann periodic structures by point defects in ionic crystals. Journal de Physique Colloques, 1980, 41 (C6), pp.C6-496-C6-499. 10.1051/jphyscol:19806129 . jpa-00220037

\section{HAL Id: jpa-00220037 https://hal.science/jpa-00220037}

Submitted on 1 Jan 1980

HAL is a multi-disciplinary open access archive for the deposit and dissemination of scientific research documents, whether they are published or not. The documents may come from teaching and research institutions in France or abroad, or from public or private research centers.
L'archive ouverte pluridisciplinaire HAL, est destinée au dépôt et à la diffusion de documents scientifiques de niveau recherche, publiés ou non, émanant des établissements d'enseignement et de recherche français ou étrangers, des laboratoires publics ou privés. 


\title{
Maxwell-Boltzmann periodic structures by point defects in ionic crystals
}

\author{
M. Georgiev \\ Institute of Solid State Physics, Bulgaran Academy of Sciences. Sofia 1184, Bulgarıa \\ N. Martinov and D. Ouroushev \\ Department of Solid State Physics, Faculty of Physics, Sofia University, Sofia 1126, Bulgaria
}

\begin{abstract}
Résumé. - La méthode du potentiel self-consistent des défauts ponctuels pour des gaz de Coulomb dans un cristal ionique, révèle une tendance à une formation de structures de défauts, semblable à celle des cristaux, avec des périodes proportionnelles à la longueur de Debye et aux structures de Maxwell-Boltzmann. L'étude thermodynamique montre qu'une solution solide de défauts chargés peut se transformer en une structure de MaxwellBoltzmann sous une température déterminée. On considère que les structures de Maxwell-Boltzmann peuvent apparaître comme des précurseurs aux états intermédiaires ou finals au cours de la désagrégation de la solution solide.
\end{abstract}

\begin{abstract}
The self-consistent-potential approach to the Coulomb gas of point defects in an ionic crystal reveals a tendency for the formation of crystal-like structures by defects with spatial periods proportional to the Debye length, Maxwell-Boltzmann structures. A thermodynamic investigation further shows that a solid solution of charged defects can convert to a Maxwell-Boltzmann structure below some realistic temperature. It is believed that Maxwell-Boltzmann structures can occur as precursors to intermediate or final states in the decay of the solid solution.
\end{abstract}

Recent theoretical studies have indicated that there is a tendency for the formation of periodic structures in a Coulomb gas [14]. As a result, the charged particles may not always distribute uniformly within the bulk but may also arrange periodically giving rise to crystal-like structures. In this way the Coulomb gas contains the genetic code for the formation of a crystal. Although this conclusion is based on the self-consistent-potential approach, it is believed to have a more general significance.

The approach to the Coulomb gas in thermal equilibrium assumes Boltzmannian energy distribution of the charged particles in a self-consistent potential field $\varphi$,

$$
n_{ \pm}=n_{0} \exp \left(\mp e \varphi / k_{\mathrm{B}} T\right)=n_{0} \exp (\mp \psi)
$$

where $\psi=e \varphi / \theta, \theta=k_{\mathrm{B}} T$. This induces a spacecharge

$$
\rho=e\left(n_{+}-n_{-}\right)=-2 e n_{0} \sinh \psi .
$$

Generally, a non-vanishing $\rho$ would result from a non-uniform spatial distribution of the particles which follows through (1) from the spatial dependence of $\varphi=\varphi(x, y, z)$. The latter is found as a solution to the Poisson-Boltzmann equation

$$
\begin{aligned}
\Delta \psi & =\varkappa^{2} \sinh \psi \\
\varkappa^{2} & =8 \pi n_{0} e^{2} / \varepsilon \theta
\end{aligned}
$$

which is obtained by inserting (2) into the Poisson equation $\Delta \varphi=-(4 \pi e / \varepsilon) \rho$. At small deviations from electrical neutrality (small $\rho$ ), $\psi \ll 1, \sin \psi \approx \psi$, consequently

$$
\Delta \psi=\varkappa^{2} \psi
$$

which is better known as the Debye equation. The simplified equation (4) has been solved under various conditions to account for different physical situations $[5,6]$ and improved solutions later found based on the complete equation $[7,8]$. However, mere improvements of the theory has not been the only result from considering the complete Poisson-Boltzmann equation which shows additional solutions with far-reaching implications [1-4].

To begin with we shall take up the one-dimensional case $[3,4]$ where $\psi=\psi(x)$. The self-consistent equipotential surfaces are now planes parallel to the $y z$-plane. Equation (3) turns into

$$
\mathrm{d}^{2} \psi / \mathrm{d} x^{2}=\varkappa^{2} \sinh \psi .
$$

A first integration of (5) leads easily to

$$
(\mathrm{d} \psi / \mathrm{d} x)^{2}=2 \chi^{2}(\cosh \psi+C)
$$

where $C$ is the integration constant. For $|C| \leqslant 1$ we set $C=1-2 k^{2}, 0 \leqslant k \leqslant 1$ and a second integration yields

$\psi(x, k)=\ln \{\operatorname{cn}(x x / 2+\beta, k) / \operatorname{sn}(x x / 2+\beta, k) \times$
$\times \operatorname{dn}(\varkappa x / 2+\beta, k)\}^{2}$ 
where $\beta$ is the second integration constant, while $\mathrm{cn}, \mathrm{sn}, \mathrm{dn}$ are the Jacobi elliptic functions [9]. These functions are periodic in $x x$ for $0 \leqslant k<1$ with periods $P(k)=4 K(k)$ where $K(k)$ is the complete elliptic integral of first kind

$$
K(k)=\int_{0}^{\pi / 2} \mathrm{~d} \alpha /\left(1-k^{2} \sin ^{2} \alpha\right)^{1 / 2}
$$

$k$ is the elliptic integrals' modulus. At $k=0$ the resulting periodicity of $\psi$ is more apparent since now cn, sn turn into the trigonometric cosine and sine respectively and $\mathrm{dn}=1$ :

$$
\psi(x, 0)=\ln \{\operatorname{cotan}(x x / 2+\beta)\}^{2} .
$$

The solution at $k=1$ is non-periodic, given by

$$
\psi(x, 1)=\ln \{\operatorname{cotanh}(x x / 2+\beta)\}^{2} .
$$

Equation (7) has a deep physical implication, for, once the self-consistent potential is periodic in $x x$, so are the particle concentrations. As a matter of fact, the spatial distributions (1) now form a periodic structure composed of alternate accumulations of positive and negative charges with a continuous distribution of charge from one accumulation point to the other. The resulting variation of $\rho$ is in the form of space-charge waves [4]. All these distributions have periods along $x$ given by

$$
p(k)=4 K(k) / x .
$$

Although solving the three-dimensional problem is not as easy, there is little doubt of the existence of periodic solutions to equation (3). As a matter of fact, one can easily construct a plane-symmetry periodic solution similar to (7) by introducing a new variable $u=x+y+z$ along the body-diagonal of the cube and assuming $\psi=\psi(u)$. Although far more difficult to find, three-dimensional periodic solutions of higher symmetry are expected to exist too. Of particular interest would be solutions having the symmetry of a crystal lattice. The corresponding periodic structures would resemble actual crystals except that there would be much more particles in an unit cell and a continuum of particles between lattice sites.

Although purely mathematical so far, the above results reveal a tendency for periodic arrangement of the charged particles. Whether or not it will materialize can be clarified by statistical thermodynamics. We have used the following procedure [2-4] : First, the free energy $G$ of the Coulomb gas in thermal equilibrium is expressed as a functional of $\psi$. Obviously $G[\psi]$ would correspond to a random distribution at $\psi=0$ and eventually to a periodic arrangement at finite $\psi$. Next, the chemical equilibrium is investigated between $G[\psi]$ and $G[0]$ to find the temperature at which the random distribution condenses into a periodic arrangement.
To illustrate this we take up the case of the Coulomb gas of divalent substitutional impurity ions $\left(n_{+}\right)$and cation vacancies $\left(n_{-}\right)$in an alkali halide crystal. The bulk free energy is

$$
\begin{aligned}
G[n] & =\int \mathrm{d} x \mathrm{~d} y \mathrm{~d} z\left\{G^{-} n_{-}+\frac{1}{2} \rho \varphi+\right. \\
& \left.+\theta\left[n_{+}\left(\ln \frac{n_{+}}{N}-1\right)+n_{-}\left(\ln \frac{n_{-}}{N}-1\right)\right]\right\}
\end{aligned}
$$

where $G^{-}$stands for the formation free energy of the vacancy, $N$ is the density of anion-cation site pairs. The particle concentrations at thermal equilibrium can be found by varying $G$ under the impurity-conservation condition

$$
\int \mathrm{d} x \mathrm{~d} y \mathrm{~d} z n_{+}=N V \bar{C}_{1}
$$

where $V$ is the integration volume, $\bar{C}_{i}$ - the average fractional concentration of (free) impurities. The result is

$$
\begin{aligned}
& n_{+}=N \exp \{-(\gamma+e \varphi) / \theta\} \\
& n_{-}=N \exp \left\{-\left(G^{-}-e \varphi\right) / \theta\right\}
\end{aligned}
$$

Equations (14) become formally identical to (1) setting

$$
\begin{aligned}
\psi & =(e / \theta)\left\{\varphi-\left(G^{-}-\gamma\right) / 2 e\right\} \\
n_{0} & =N \exp \left\{-\left(G^{-}+\gamma\right) / 2 \theta\right\} .
\end{aligned}
$$

The Lagrange multiplier $\gamma$ can be found inserting (14) into (13):

$$
\begin{aligned}
\gamma=-\left\{G^{-}+2 \theta \ln \left(\bar{C}_{\mathrm{i}} V / \int \mathrm{d} x \mathrm{~d} y \mathrm{~d} z \times\right.\right. \\
\times \exp (-\psi))\} .
\end{aligned}
$$

It can be seen that $\gamma$ depends on the spatial distribution of the charged particles. For a random distribution $(\psi=0)$ :

$$
\gamma_{\mathrm{R}}=-\left(G^{-}+2 \theta \ln \bar{C}_{\mathrm{i}}\right) .
$$

Deriving $\gamma_{P}$ for a periodic arrangement is not straightforward.

Inserting back (1) into (12) using (15) one obtains

$$
G[\psi]=-\left\{(\gamma+2 \theta) / 2+E_{1}\right\}\left(N_{+}+N_{-}\right)
$$

where the following notations are used :

$$
N_{ \pm}=n_{0} \int \mathrm{d} x \mathrm{~d} y \mathrm{~d} z \exp (\mp \psi)
$$

the total number of particles of either sign and

$$
E=E_{1}\left(N_{+}+N_{-}\right)=-n_{0} \theta \int \mathrm{d} x \mathrm{~d} y \mathrm{~d} z \psi \sinh \psi
$$


$E$ has the meaning of a total (electrostatic) binding energy which follows from (20) and (2). In all the above manipulations the in tegration volume has been assumed electrically neutral

$$
\int \mathrm{d} x \mathrm{~d} y \mathrm{~d} z \sinh \psi \alpha \int \mathrm{d} x \mathrm{~d} y \mathrm{~d} z \rho=0 .
$$

Equation (18) will be investigated separately for random and periodic distributions. In the former case

$$
\begin{aligned}
G[0] & =-\frac{1}{2}\left(\gamma_{\mathrm{R}}+2 \theta\right)\left(N_{+}+N_{-}\right)= \\
& =\frac{1}{2}\left(G^{-}-2 \theta+2 \theta \ln \bar{C}_{\mathrm{i}}\right)\left(N_{+}+N_{-}\right) .
\end{aligned}
$$

For the latter we note that the one-particle energy $E_{1}$ depends on the symmetry of the periodic solution. For plane-symmetry we have from (5), (6) and the periodicity of $\psi$ :

$$
E=\theta\left\{N_{+}+N_{-}+2\left(1-2 k^{2}\right) N_{0}\right\} .
$$

Inasmuch as $N_{0}=n_{0} V<N_{ \pm}$we practically have $E_{1}=\theta$. For cubic symmetry the solutions are unknown but we have tentatively used the Madelung energy $E_{\mathrm{M}}=\alpha_{\mathrm{M}} e^{2} / 2 \varepsilon d$ for $E_{1}[2,3]$.

From (21) and (18) we can find the equilibrium between the random and periodic distributions. Equating the chemical potentials of the particles we have $\gamma_{\mathrm{R}}=\gamma_{\mathrm{P}}+2 E_{1}$ which yields

$$
\bar{C}_{\mathrm{i}}=\exp \left\{-\left(G^{-}+\gamma_{\mathrm{P}}+2 E_{1}\right) / 2 \theta\right\} .
$$

Equation (23) gives the saturated free impurity concentration in the random phase in equilibrium with the periodic structure.

$\gamma_{p}$ should be derived strictly by means of the variational principle. This has been done for the planesymmetry case leading to extremal spatial periods of $\sim 1000 \AA$ at $300 \mathrm{~K}$ [4]. In another instance we have instead made use of the relationship between $\gamma_{P}$ and the statistical period $p$, which can be presented in a more general form : $p=4 Q / x$, where $Q$ is an unknown parameter, to include also symmetries other than plane-like. From (3) and (15) :

$$
\begin{aligned}
& \gamma_{\mathrm{p}}=-\left(G^{-}+2 \theta \ln \left(\theta / \theta_{\mathrm{q}}\right)\right) \\
& \theta_{\mathrm{q}}=\pi N e^{2} p^{2} / 2 \varepsilon Q^{2} .
\end{aligned}
$$

Inserting into (23) we find

$$
\bar{C}_{\mathrm{i}}=\left(\theta / \theta_{\mathrm{q}}\right) \exp \left(-E_{1} / \theta\right)
$$

Note that the pre-exponential factor arises from the statistical interpretation of the spatial period. $\bar{C}_{i}$ in (25) can be regarded as activity rather than concentration. Conventional theories of activity lead to constant pre-exponential factor, while the experiment often gives a factor that increases with temperature [10]. Inasmuch as equation (25) is of the latter type, we tried to fit it to experimental data on the dissolution of the Suzuki phase, such as $6 \mathrm{NaCl} . \mathrm{MnCl}_{2}$ and $6 \mathrm{NaCl} \mathrm{CdCl}_{2}$ [10]. The result was encouraging, particularly in the $\mathrm{Mn}$ case, where reasonable values for $E_{1}$ and $\theta_{\mathrm{q}}$ were obtained from the least squares' fit. While noone would completely identify continuous statistical structures with discrete superlattices, the observed similarity raises the important question as to whether the mechanism which triggers the formation of the Suzuki superlattice is not of statistical nature.

We propose to call the present continuum periodic arrangements Maxwell-Boltzmann structures to underline their statistical nature. They follow from the balance between diffusion and electric currents in a Coulomb gas and are as intrinsic to thermal equilibrium as, say, the Debye atmosphere surrounding each ion. In a sense the MB structures can be regarded as a particular case of a screening profile in which the charged particles arrange periodically and alternately relative to the central ion.

Although no experimental observations have been reported so far of $\mathrm{MB}$ structures, they can undoubtedly influence a number of physical properties of matrix media such as light scattering, thermal and electric conduction (new knees on the ionic conductivitytemperature curves), dielectric losses, etc. They can cause light diffraction or induce changes in the surface states of crystals. Their most likely occurrence seems to be in solid electrolytes appearing as precursors to final or intermediate states in the decay of a solid solution.

Acknowledgement. - One of the authors (M. G.) is greatly indebted to the British Council and the Organizers for a grant which made possible his participation in the Conference.

\section{DISCUSSION}

\section{Question. - A. B. Lidiard.}

Your observation that the Maxwell-Boltzmann equation admits periodic solutions is very interesting. Can you give examples to show the conditions under which these periodic solutions correspond to the lowest free energy?

\section{Reply. - M. GEORGIEv.}

For instance, we have calculated the conversion temperature to be the order of $300-400 \mathrm{~K}$ at an impurity concentration of about $10^{-4} \mathrm{ppm}$, for the transformation of the random defect distribution into a plane-symmetry periodic structure. 
Question. - R. J. Friaur.

What are the factors that determine the periodicity length in your theory? Also could you give some numerical values for a typical case.

\section{Reply. - M. GeORGIEv.}

The periodicity length depends on the bulk impurityconcentration, as well as on the temperature. For a plane-symmetry $\mathrm{MB}$ structure we obtain periods of the order of $1000 \AA$ at room temperature.

\section{References}

[1] Georgiev, M. Martinov, N., Ouroushev, D., Acta Crustallogr. Suppl. A 34 (1978)

[2] Georgiev, M., Martinov, N., Ouroushev, D., Solid State Commun. 28 (1978) 779.

[3] Martinov, N., Georgiev, M., Ouroushev, D., Bulg. J. Phys. 6 (1979) 38 .

[4] Grorgiev, M., Martinov, N., Ouroushev, D., Crystal Lattice Deferts. in press (1979).

[5] Moel.wyn-Hughes, E. A., Physical Chemistry (Pergamon Press, London) 1961.
[6] Eshelby, J. D., Newey, C. W. A., Pratt, P. L., Lidiari). A. B., Phil. Mag. 3 (1958) 75.

[7] Koghler, J. S., Langreth, D., Turkovich, B. v., Phys. Rev. 128 (1962) 573.

[8] Kligwer, K. L., Koehler, J. S., Phys. Rev. 140 (1965) A 1226.

[9] JANKE, E., EMDE, F., LösCH, F., Tafeln höherer Funktionen (Teubner, Stuttgart) 1960.

[10] Chapman, J. A., Llliey, E., J. Physique Colloq. 34 (1973) C9-341. 